\title{
B-physics anomalies, fluctuations and patterns: a status report
}

\section{Sébastien Descotes-Genon*,}

Laboratoire de Physique Théorique (UMR8627),

CNRS, Univ. Paris-Sud, Université Paris-Saclay, 91405 Orsay, France

E-mail: descoteseth.u-psud.fr

I review the current status of the $B$ anomalies in $b \rightarrow c \ell \bar{v}_{\ell}$ and $b \rightarrow s \ell \ell$ transitions. After recalling the experimental situation in each case, I discuss the elements of the Standard Model (SM) predictions and the patterns of New Physics arising in model-independent analyses, before discussing some of the models beyond the SM proposed to explain these patterns.

ALPS 2019 An Alpine LHC Physics Summit April 22 - 27, 2019

Obergurg, Austria

${ }^{*}$ Speaker. 


\section{Introduction}

Due to the structure of the Standard Model (SM), some quark flavour transitions are expected to be particularly sensitive to intermediate states with high energy scales and possibly involving New Physics (NP) degrees of freedom. The presence of widely different scales (non-perturbative QCD, heavy-quark masses, electroweak interaction, NP) makes the computation of such processes a tough theoretical challenge. It can be tackled using a ladder of effective field theories to separate the different dynamical scales involved: most can be treated perturbatively, leaving only non-perturbative inputs involving long-distance QCD hadronisation effects.

Recent measurements from the LHCb, Babar and Belle experiments suggest several interesting deviations from the SM in such quark transitions. The first set of deviations occurs in $b \rightarrow c \ell \bar{v}_{\ell}$, by comparing branching ratios involving $\tau$ and lighter leptons, whereas the second happens for $b \rightarrow s \ell \ell$, in muon observables and in ratios of branching ratios for electron and muon modes. The latter decay is suppressed in the SM and is expected to show sensitivity to NP effects, whereas the former is a tree-level transition in the SM and is less expected to show large deviations due to higher-energy phenomena. Interestingly, both channels suggest NP effects violating leptonflavour universality, an unambiguous sign of NP [1], even though recent results from Belle tend to reduce the discrepancy with respect to the SM observed by LHCb. Their description requires a careful assessment of the theoretical uncertainties of the SM predictions, and these deviations can be studied either in model-dependent or model-independent approaches to NP.

\section{2. $b \rightarrow c \ell v$ transitions}

\subsection{Experimental situation and SM predictions}

The transitions $b \rightarrow c \ell \bar{v}_{\ell}$, where $\ell=e, \mu, \tau$ have been studied at LHCb, Babar and Belle (with different $\tau$ identification techniques for LHCb and B-factories) [2]. It turns out that the ratios $R(D)$ and $R\left(D^{*}\right)$ exceed SM predictions by $1.4 \sigma$ and $2.5 \sigma$ respectively, leading to a combined discrepancy with SM at $3.1 \sigma$ (see Fig. 1):

$$
R_{D(*)}=\frac{B r\left(B \rightarrow D\left(^{*}\right) \tau \nu\right)}{B r\left(B \rightarrow D\left({ }^{*}\right) \ell \bar{v}_{\ell}\right)} .
$$

The individual branching ratios are consistent with a $10 \%$ enhancement for $b \rightarrow c \tau \bar{v}_{\tau}$ compared to the SM expectations. Deviations from lepton-flavour universality have also been observed in the branching ratios of $B_{c} \rightarrow J / \psi \ell \bar{v}_{\ell}$ (comparing again light and heavy leptons), but the SM prediction is affected by significant uncertainties. The Belle collaboration has also measured the $\tau$ and $D^{*}$ polarisations (the latter with a very good precision) which are both compatible with the SM.

The SM prediction for the $B \rightarrow D \ell \bar{v}_{\ell}$ branching ratio involves two hadronic inputs: a vector form factor and, in the case of $\ell=\tau$, a scalar one (a generic extension of the SM would require a third, tensor, form factor). These form factors can be computed through lattice QCD (LQCD) simulations [3]. The LQCD results for the vector form factor show a dependence on the leptonpair invariant mass $q^{2}$ in good agreement with the Babar measurement for $\ell=e, \mu . B \rightarrow D^{*} \ell \bar{v}_{\ell}$ proves more challenging [4]: four different form factors are needed for the SM predictions (and 3 more, tensor, form factors would be needed for a generic NP model). There is no complete lattice 

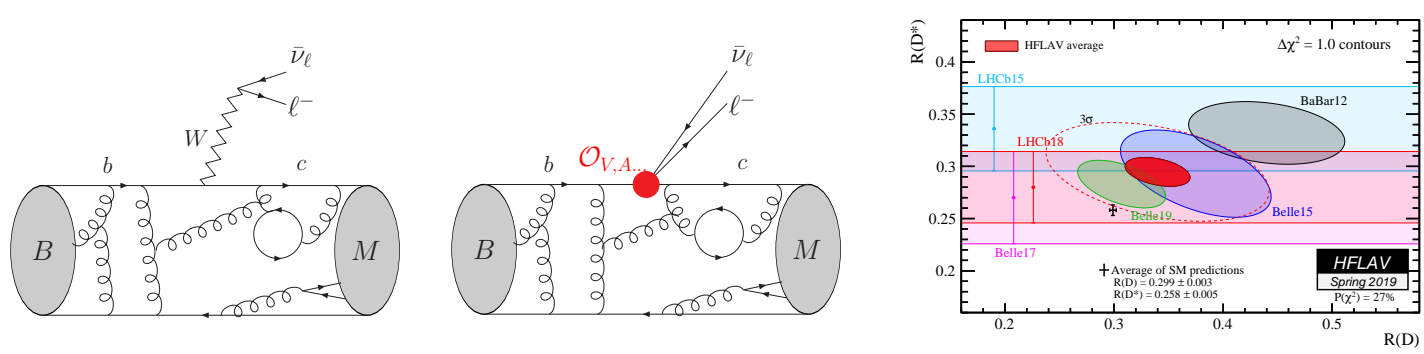

Figure 1: From left to right: SM diagram for $B \rightarrow D\left(^{*}\right) \ell \bar{v}_{\ell}$ transitions, corresponding description in the effective Hamiltonian framework, and experimental situation concerning the ratios of branching ratios $R(D)$ and $R\left(D^{*}\right)$ (HFLAV average in red) compared to the SM prediction (cross).

determination for the $B \rightarrow D^{*}$ form factors, leading to the use of Heavy Quark Effective Theory: the form factors can be related in the heavy-quark limit $m_{b} \rightarrow \infty$, providing ratios of form factors up to $O\left(\Lambda / m_{B}\right)$ corrections. A normalisation is provided by Belle results for $B \rightarrow D^{*} \ell \bar{v}_{\ell}(\ell=e, \mu)$ assuming that there is no NP involved in the case of light leptons. Part of the hadronic uncertainties stemming from the form factors cancel in the ratios $R_{\left.D^{*}\right)}$, but not completely, which makes a lattice estimation of the whole set of form factors very important to confirm the deviation in these ratios.

In order to make progress on this issue, additional $B \rightarrow D^{*} \ell \bar{v}_{\ell}$ observables can be built from the geometry of the decay products and the comparison of different lepton modes [5]. Other interesting perspectives consist in checking the $q^{2}$-dependence of the differential decay rates for both vector and pseudoscalar final mesons, or considering baryon modes [6].

\subsection{Interpretation through an effective Hamiltonian approach}

The deviations can be easily interpreted by adding new interactions to the effective Hamiltonian describing FCCC at the scale of the $b$-quark mass, integrating out heavier degrees of freedom:

$$
\begin{aligned}
\mathscr{H}^{\mathrm{eff}}=2 \sqrt{2} G_{F} V_{c b} \sum_{\ell=e, \mu, \tau}\left[\left(1+\mathscr{C}_{V \ell}^{L}\right)\left(\bar{c} \gamma^{\mu} P_{L} b\right)\left(\bar{\ell} \gamma^{\mu} P_{L} v_{\ell}\right)\right. \\
+\mathscr{C}_{S \ell}^{R}\left(\bar{c} P_{R} b\right)\left(\bar{\ell} P_{L} v_{\ell}\right)+\mathscr{C}_{S \ell}^{L}\left(\bar{c} P_{L} b\right)\left(\bar{\ell} P_{L} v_{\ell}\right)+\mathscr{C}_{T \ell}\left(\bar{c} \sigma^{\mu v} P_{L} b\right)\left(\bar{\ell} \sigma_{\mu v} P_{L} v_{\ell}\right)
\end{aligned}
$$

with $P_{L, R}=\left(1 \mp \gamma_{5}\right) / 2$ and the first line corresponds to the SM $V-A$ interaction. The deviations observed can then be easily interpreted as non-vanishing values of the Wilson coefficients $\mathscr{C}_{i}$. In addition to the constraints from $B \rightarrow D\left(^{*}\right) \ell \bar{v}_{\ell}$, the $B_{c}$ lifetime provides tight bounds for righthanded and scalar couplings which contribute significantly to $B_{c} \rightarrow \tau \nu$ [7]. Several scenarios of contributions from two different $\tau$ operators, often suggested by NP models, can explain the patterns observed. But the most economical explanation consists in assuming a single contribution to $\mathscr{C}_{V \tau}^{L}$, i.e. a NP modification of the Fermi constant for $b \rightarrow c \tau v$ transition, affecting the branching ratios but not the angular observables (see Refs. [8] and references therein).

\section{3. $b \rightarrow s \ell \ell$ transitions}

\subsection{Experimental situation and effective Hamiltonian approach}

The transitions $b \rightarrow s \ell^{+} \ell^{-}$are studied in detail in the LHCb, CMS and ATLAS experiments, as 

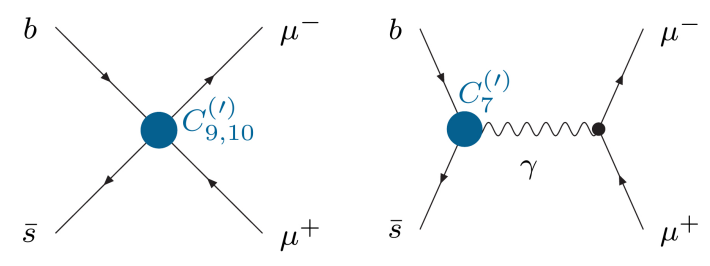

\begin{tabular}{c|c|c|c} 
processes & $\mathscr{C}_{7\left({ }^{\prime}\right)}$ & $\mathscr{C}_{9\left(^{\prime}\right) \mu}$ & $\mathscr{C}_{10\left(^{\prime}\right) \mu}$ \\
\hline \hline$B \rightarrow X_{s} \gamma, B \rightarrow K^{*} \gamma$ & $\mathrm{x}$ & & \\
\hline$B \rightarrow X_{s} \mu^{+} \mu^{-}$ & $\mathrm{x}$ & $\mathrm{x}$ & $\mathrm{x}$ \\
\hline$B_{s} \rightarrow \mu^{+} \mu^{-}$ & & & $\mathrm{x}$ \\
\hline$B_{(s)} \rightarrow\left(K^{(*)}, \phi\right) \mu^{+} \mu^{-}$ & $\mathrm{x}$ & $\mathrm{x}$ & $\mathrm{x}$ \\
\hline
\end{tabular}

Figure 2: Effective couplings $\mathscr{C}_{7\left({ }^{\prime}\right), 9\left(^{\prime}\right) \mu, 10\left(^{\prime}\right) \mu}$ contributing to $b \rightarrow s \mu \mu$ transitions and sensitivity of the various radiative and (semi)leptonic $B_{(s)}$ decays to them.

well as in the Belle and Belle II collaborations. Recent experimental results have shown interesting deviations from the SM [1]. Branching ratios for $B \rightarrow K\left(^{*}\right) \mu \mu, B_{s} \rightarrow \phi \mu \mu$ are found consistently lower than the SM expectations over the whole kinematic range, whereas deviations were observed for some of the $B \rightarrow K^{*} \mu^{+} \mu^{-}$angular asymmetries built specifically to yield clean theoretical predictions, namely the angular observable $P_{5}^{\prime}[9]$ at large $K^{*}$ recoil. The ratios

$$
R_{K(*)}=\frac{B r\left(B \rightarrow K\left(^{*}\right) \mu^{+} \mu^{-}\right)}{B r\left(B \rightarrow K\left({ }^{*}\right) e^{+} e^{-}\right)}
$$

were measured by LHCb showing deviations from SM expectations between 2.3 and $2.5 \sigma$, whereas Belle measured recently the same observables with larger uncertainties, leading to results in agreement with both the SM and LHCb measurements. These deviations from lepton flavour universality seem related to anomalies in $b \rightarrow s \mu \mu$ transitions, since there are no indications of deviations from the SM in the Belle and LHCb measurements for $b \rightarrow$ see branching ratios and angular observables (although these measurements are challenging and thus with larger uncertainties than for $b \rightarrow s \mu \mu$ ). Other observables, probing different aspects of the $b \rightarrow s \mu \mu$ transition, are in good agreement with SM expectations, for instance the branching ratios for $B_{s} \rightarrow \mu \mu$ or the inclusive $B \rightarrow X_{s} \gamma$ decay.

The appearance of several tensions in different $b \rightarrow s \mu \mu$ channels is interesting since all the channels are dominantly sensitive to the same couplings $\mathscr{C}_{7\left(^{\prime}\right), 9\left(^{\prime}\right) \ell, 10\left(^{\prime}\right) \ell}$ induced by the operators in the effective Hamiltonian approach (see Fig. 2)

$$
\begin{array}{lll}
\mathscr{O}_{9\left(^{\prime}\right) \ell}=\frac{\alpha}{4 \pi}\left[\bar{s} \gamma^{\mu} P_{L(R)} b\right]\left[\bar{\mu} \gamma_{\mu} \mu\right], & \mathscr{O}_{10\left(^{\prime}\right) \ell}=\frac{\alpha}{4 \pi}\left[\bar{s} \gamma^{\mu} P_{L(R)} b\right]\left[\bar{\mu} \gamma_{\mu} \gamma_{5} \mu\right], & \mathscr{O}_{7\left(^{\prime}\right)}=\frac{\alpha}{4 \pi} m_{b}\left[\bar{s} \sigma_{\mu v} P_{R(L)} b\right] F^{\mu v}, \\
\mathscr{C}_{9 \ell}^{\mathrm{SM}}\left(\mu_{b}\right)=4.07, & \mathscr{C}_{10 \ell}^{\mathrm{SM}}\left(\mu_{b}\right)=-4.31, & \mathscr{C}_{7}^{\mathrm{SM}}\left(\mu_{b}\right)=-0.29,
\end{array}
$$

where $m_{b}$ denotes the $b$ quark mass and $\mu_{b}=4.8 \mathrm{GeV}$. Primed operators have vanishing or negligible Wilson coefficients in the SM, whereas the SM operators with the same structure but different lepton flavours have identical Wilson coefficients in the SM due to lepton-flavour universality. It is natural to ask whether a NP contribution to these couplings could account for all the tensions at once, coming from particular extensions of the SM inducing contributions to some Wilson coefficients. The couplings $\mathscr{C}_{7,9,10}^{(\prime)}$ can be constrained through various observables in radiative and (semi) leptonic $B_{(s)}$ decays, each of them sensitive to a different subset of coefficients (see Fig. 2).

\subsection{Hadronic uncertainties}

At leading order (LO) in the effective theory, SM predictions involve tree-level diagrams with insertions of the operators $\mathscr{O}_{7,9,10}$ (generated at one loop in the SM), as well as one-loop diagrams 

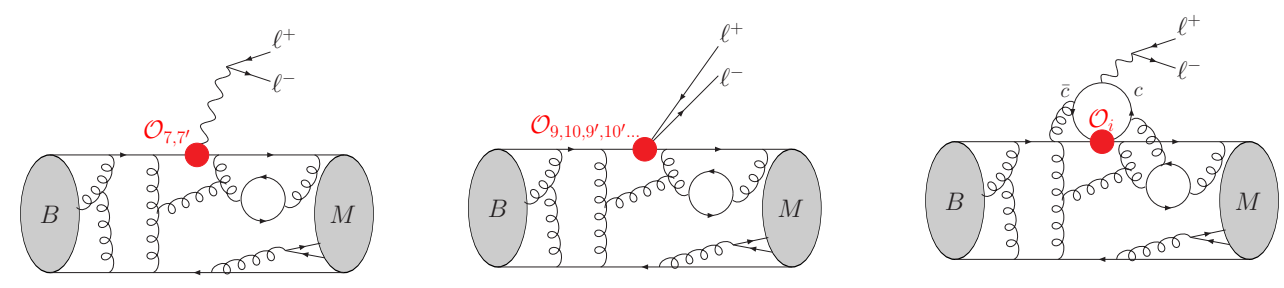

Figure 3: Illustration of local (first two diagrams) and non-local (third diagram) contributions to exclusive $B \rightarrow M \ell^{+} \ell^{-}$matrix elements.

with an insertion of the charged-current operator $\mathscr{O}_{2}=\left[\bar{s} \gamma^{\mu} P_{L} c\right]\left[\bar{c} \gamma_{\mu} P_{L} b\right]$ (generated at tree level in the SM). In the first case the leptonic and the hadronic currents factorise, QCD corrections are restrained to the hadronic $B \rightarrow M$ current (first two diagrams in Fig. 3), leading to hadronic form factors parametrising the $B \rightarrow M$ transition. Contributions of the second type exhibit a non-local structure related to charm loops (third diagram in Fig. 3) that cannot be absorbed into form factors and that can become large when the $c \bar{c}$ pair becomes resonant (i.e. when the dilepton invariant mass becomes close to one of the charmonium resonances).

A first source of theoretical uncertainties comes from form factors, which are available from LQCD as well as from light-cone sum rule (LCSR) calculations, with the former being suited for the region of high $q^{2}>15 \mathrm{GeV}^{2}$ (low $K^{*}$ recoil) and the latter for the region of low $q^{2}<$ $8 \mathrm{GeV}^{2}$ (large $K^{*}$ recoil) Several determinations from light-cone sum rules have been proposed, using different formulations $[10,11]$. Some of these determinations have also been combined with LQCD results to provide a parametrisation over the whole kinematic range, with very small error bars. However, these sum rules rely often on specific assumptions $\left(K^{*}\right.$ treated as an infinitely narrow resonance, contributions from higher excitations and/or higher-twist distribution amplitudes neglected) which should be investigated to attach a systematic uncertainty to these hypotheses [12].

Since the form factors introduce an important source of theoretical uncertainties and can be affected by additional systematics to be investigated, it is desirable to reduce the sensitivity of the predictions to these inputs as much as possible. For $B \rightarrow K^{*} \ell^{+} \ell^{-}$decays, with $K^{*}$ being a vector meson, this can be achieved in the low- $q^{2}$ region by exploiting large-recoil symmetries of QCD. relating the various hadronic form factors at LO in $\alpha_{s}$ and $\Lambda / m_{b}$. The coefficients of the differential angular distribution can then be used to build observables with a more limited sensitivity to the form factors by exploiting these relationships [14, 9, 13], and the resulting optimised observables $P_{i}^{(')}$ at large recoil exhibit a mild form factor dependence, suppressed by powers of $\alpha_{s}$ and $\Lambda / m_{b}$.

A second source of uncertainties come from $c \bar{c}$ loops. Long-distance charm-loop effects (third diagram in Fig. 3) can mimic the effect of an effective coupling $\mathscr{C}_{9}^{c \bar{c}}$. However, they have rather specific features, as their contribution is expected to have a non-constant $q^{2}$-dependence, where $q^{2}$ is the invariant mass of the lepton pair, and to be process dependent. Together with the perturbative SM contribution $\mathscr{C}_{9}^{\text {eff }}$ SMert and a potential constant NP coupling $\mathscr{C}_{9}^{\mathrm{NP}}$, it can be cast into an effective Wilson coefficient $\mathscr{C}_{9}^{\text {eff i }}\left(q^{2}\right)=\mathscr{C}_{9 \mathrm{SM} \text { pert. }}^{\text {eff }}\left(q^{2}\right)+\mathscr{C}_{9}^{\mathrm{NP}}+\mathscr{C}_{9}^{c \bar{c} i}\left(q^{2}\right)$, with a different $\mathscr{C}_{9}^{c \bar{c} i}$ and hence also a different $\mathscr{C}_{9}^{\text {eff i }}$ for the three transversity amplitudes $i=0, \|, \perp$. The evaluation of this longdistance contribution is difficult, especially close to the region of charmonium resonances. Several estimates are available, based on light-cone sum rules, a sum of Breit-Wigner resonances or a 


\begin{tabular}{crccc} 
Coefficient & Best fit & $1 \sigma$ & Pull & $p$-value \\
\hline $\mathscr{C}_{9}^{\mathrm{NP}}$ & -1.02 & {$[-1.18,-0.85]$} & 5.8 & $65 \%$ \\
$\mathscr{C}_{9}^{\mathrm{NP}}=-\mathscr{C}_{10}^{\mathrm{NP}}$ & -0.49 & {$[-0.59,-0.40]$} & 5.4 & $55 \%$ \\
$\mathscr{C}_{9}^{\mathrm{NP}}=-\mathscr{C}_{9^{\prime}}^{\mathrm{NP}}$ & -1.02 & {$[-1.18,-0.85]$} & 5.7 & $61 \%$
\end{tabular}

Figure 4: Some NP scenarios favoured in the global fit to $b \rightarrow s \ell \ell$ observables.

general parametrisation relying on unitarity [10,15]. All agree well with each other and estimates for charm-loop effects are naturally included in the SM predictions for the various observables.

\subsection{Global analysis of $b \rightarrow s \ell \ell$ data}

The interest of a global analysis of various hadronic channels and observables was realised much before the advent of $\mathrm{LHCb}$ data [16]. The first analysis performed in this spirit and exploiting the LHCb 2013 data [17] pointed to a large negative contribution to the Wilson coefficient $\mathscr{C}_{9}$, as confirmed by other groups [18], and updated to take into account the recent measurements described in Sec. 3.1 [19, 20]. Starting from a model hypothesis with free parameters for some Wilson coefficients $\left\{\mathscr{C}_{i}^{\mathrm{NP}}\right\}$, we performed a frequentist fit, including experimental and theoretical correlation matrices. For each one-parameter scenarios, we could give the SM-pull for each scenario, i.e. by how many sigmas the best fit point is preferred over the SM point $\left\{\mathscr{C}_{i}^{\mathrm{NP}}\right\}=0$ in the given scenario: a scenario with a large SM-pull leads to a big improvement over the SM and a better description of the data. The $p$-value is also an interesting piece of information, indicating the quality of the fit and thus the ability for the scenario to fit the experimental data (for the SM, it amounts to 9\%). The successful scenarios needed a large negative $\mathscr{C}_{9}^{\mathrm{NP}}$ [20], as can be seen from Figs. 4 and 5, with similar results when analysing subsets of data. A full 6-parameter fit of $\left.\mathscr{C}_{7,9,10}^{(}\right) \mathrm{NP}$ results in a SM-pull of $5.3 \sigma$ and a $p$-value of $82 \%$, with only $\mathscr{C}_{9}\left(\mathscr{C}_{10}\right)$ deviating significantly (mildly) from its SM value [19]. These results illustrate that the deviations observed in $b \rightarrow s \mu \mu$ channels follow a consistent pattern, the various deviations adding up to favour simple NP scenarios corresponding to deviations in a few Wilson coefficients only.

These results agree well with our previous analyses [17, 19, 20] and with other recent global analyses involving similar sets of up-to-date data [21]. All analyses agree and prefer scenarios involving a contribution to $\mathscr{C}_{9 \mu}$ in $b \rightarrow s \mu \mu$, whereas the other Wilson coefficients are only loosely bound and compatible with SM. The hierarchy among the various NP scenarios (based on their SM-pulls) depend on specific details of the analyses and inputs, but the preferences for scenarios involving contributions to $\mathscr{C}_{10 \mu}$ and/or $\mathscr{C}_{9 \mu}$ are found in all analyses. These works also considered hadronic uncertainties (power corrections, form factors, charm-loop contributions): only very large contributions would be needed to reproduce the anomalies in $b \rightarrow$ sll data, with difficulties to account for the consistency of deviations observed in the various channels and observables.

In the fits, NP is allowed in $b \rightarrow$ see but not mandatory as for $b \rightarrow s \mu \mu$. It proves interesting to recast this question in terms of two kinds of NP, Lepton Flavour Universal (LFU) and Lepton Flavour Universality Violating (LFUV), so that the Wilson coefficients read [19]: $\mathscr{C}_{i e}^{\mathrm{NP}}=\mathscr{C}_{i}^{\mathrm{U}}$ and $\mathscr{C}_{i \mu}^{\mathrm{NP}}=\mathscr{C}_{i}^{\mathrm{U}}+\mathscr{C}_{i}^{\mathrm{V}}$ (with $i=9,10$ ) for $b \rightarrow s e^{+} e^{-}$and $b \rightarrow s \mu^{+} \mu^{-}$transitions respectively. Several 

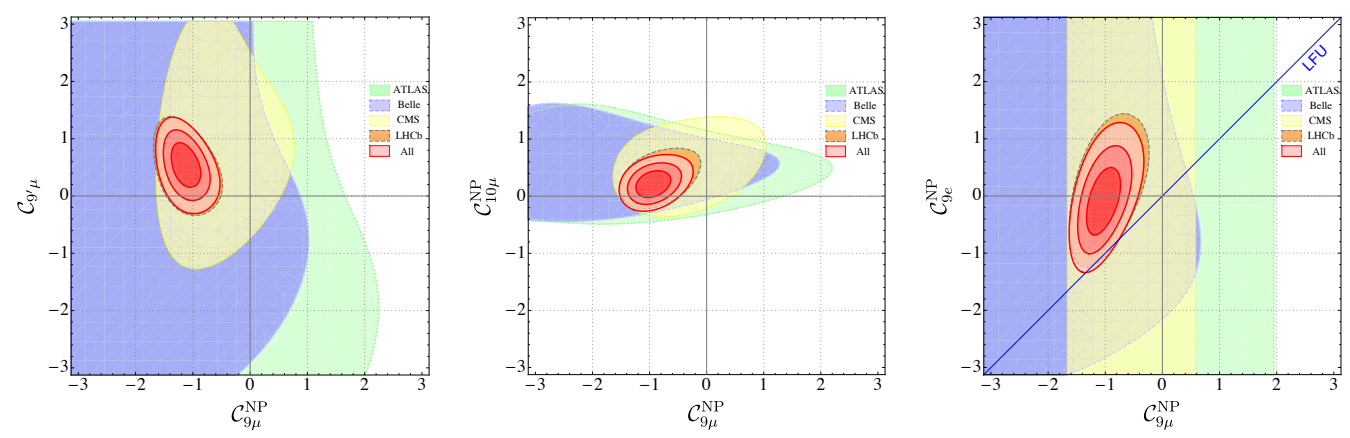

Figure 5: Results for the two-dimensional scenario with real contributions to $\mathscr{C}_{9 \mu}$ and $\mathscr{C}_{10 \mu}$ (left), $\mathscr{C}_{9 \mu}$ and $\mathscr{C}_{9^{\prime} \mu}$ (center), $\mathscr{C}_{9 \mu}$ and $\mathscr{C}_{9 e}$ (right).

NP scenarios fit very well the data, e.g. $\mathscr{C}_{9}^{\mathrm{U}}$ and $\mathscr{C}_{9}^{\mathrm{V}}=-\mathscr{C}_{10}^{\mathrm{V}}$ (SM-pull at $5.9 \sigma$ and $p$-value of $74 \%$ ) with vanishing contributions excluded at more than $2 \sigma$ for both LFU an LFUV NP.

Such LFU contributions arise naturally from radiative corrections. For instance the scenario that we have just discussed arises naturally when one tries to explain both $R_{D(*)}$ and $b \rightarrow$ sll anomalies [20] within the Standard Model Effective Field Theory. The SMEFT is the effective theory obtained at the electroweak scale assuming that NP occurs at a sufficiently high scale and appears thus as dimension-6 operators to the SM Lagrangian. An operator involving $\tau$ leptons explains $R_{D^{(*)}}$ (a similar one involving muons gives a LFUV effect in $b \rightarrow s \mu^{+} \mu^{-}$). $S U(2)_{L}$ invariance leads generally to the enhancement of $b \rightarrow s \tau^{+} \tau^{-}$processes [23], and radiative corrections generate a contribution to $\mathscr{C}_{9}^{\mathrm{U}}$ [24]. This yields a correlation between $\mathscr{C}_{9}^{\mathrm{U}}$ and $R_{D^{(*)}}$ shown on the left of Fig. 6 (see also Ref. [21]). This scenario has a pull of $7.0 \sigma$ due to the inclusion of $R_{D^{(*)}}$.

We find several NP scenarios with a similar ability to explain the deviations observed in the data (SM pull, $p$-value). A better theoretical understanding in the hadronic uncertainties (in particular charm loops) would help to disentangle these scenarios, as well as more precise measurements. More observables would also be useful, for instance angular observables assessing LFUV like $Q_{5}[13,22]$ or observables from baryon modes [25].

\section{NP models}

Since hints of LFUV are only seen in $b$-quark transitions involving leptons, it is tempting to build models explaining both $b \rightarrow c \ell \bar{v}$ and $b \rightarrow s \ell \ell$ deviations simultaneously. A framework to correlate the deviations in the tree- and loop-level $b$-quark transitions, is provided by the SMEFT. In this theory, two types of higher-dimension operators (scalar and tensor) are involved in the explanation of the anomalies. Under fairly general assumptions concerning the flavour structure of these operators, one can parametrise the contributions according to two couplings $C_{S}$ and $C_{T}$ that can be fitted to the data for $b \rightarrow c \ell \bar{v}$ and $b \rightarrow s \ell \ell$ channels as well as other flavour constraints [26].

The result is shown on the right of Fig. 6 together with lines corresponding to simplified models involving a single mediator. Colourless vectors do not seem to be able to accommodate all the data $\left(B_{s} \bar{B}_{s}\right.$ mixing, direct production at the LHC). Scalar leptoquarks may in principle provide a good fit to the data, but the radiative corrections to $Z \rightarrow t \bar{t}$ and $Z \rightarrow v \bar{v}$ are significant and create 

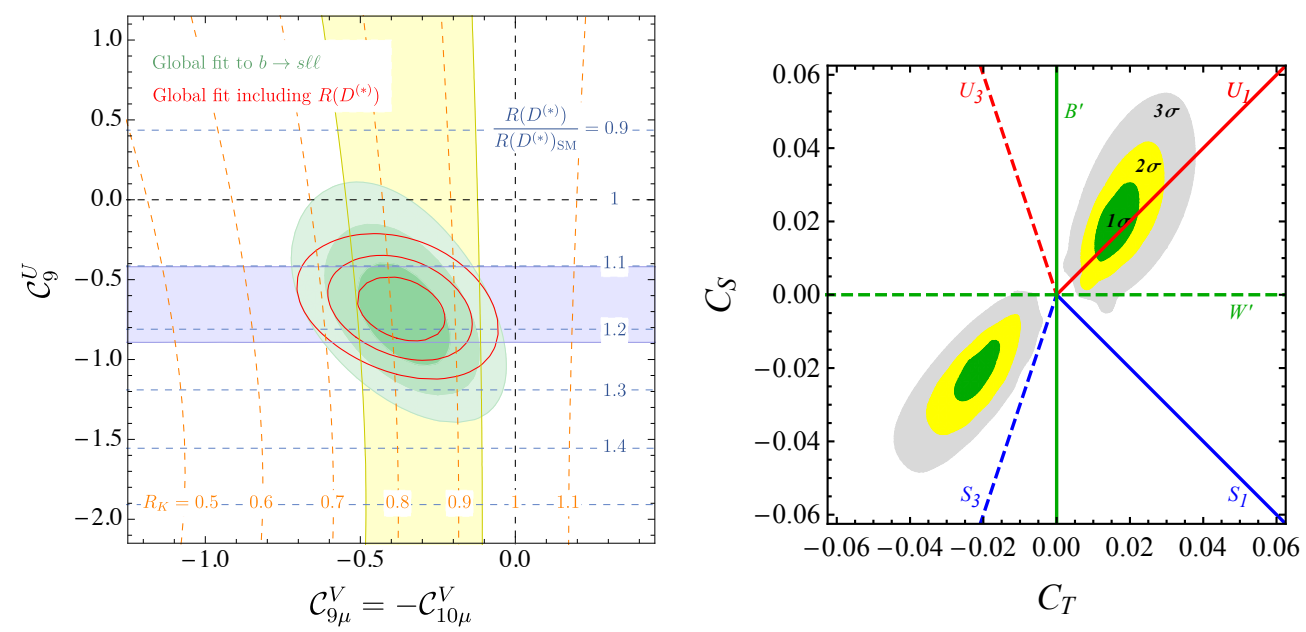

Figure 6: Left: constraints of the scenario with LFU contribution $\mathscr{C}_{9}^{\mathrm{U}}$ and LFUV contribution $\mathscr{C}_{9}^{\mathrm{V}}=-\mathscr{C}_{10}^{\mathrm{V}}$ in an EFT framework connecting $b \rightarrow c \ell v$ and $b \rightarrow s \ell v$. Right: interpretation in terms of single-mediator models for the data on $b \rightarrow c \ell v$ and $b \rightarrow s \ell \ell$, where the coloured regions correspond to a fit to flavour data and the lines to single-mediator models.

a mild tension with $R_{D^{*}}$. In both cases, these problems can be solved by including further fields in the theory. Finally, the vector leptoquark singlet $U_{1}$ fit very well the data. Such a massive vector leptoquark requires additional fields to build an ultraviolet complete model, for which several possibilities have been investigated further, such as Pati-Salam models or partial compositeness. These models feature additional particles, such as heavy vector-like quarks and leptoquarks.

\section{Conclusions}

$b$-quark decays exhibit interesting patterns of deviations with respect to SM predictions in two different sectors: the tree-level charged-current transitions $b \rightarrow c \ell \bar{v}_{\ell}$ and the loop-level neutralcurrent processes $b \rightarrow s \ell \ell$. In the first case, a few observables are available (branching ratios) whereas in the latter case, many quantities (branching ratios, angular observables) are measured. Interestingly, both channels suggest NP contributions distinguishing between the various leptons. Fits to additional NP couplings within the effective Hamiltonian approach can be performed. Even though hadronic uncertainties play a significant role here and should be assessed more precisely in the future, NP scenarios related to consistent patterns of deviations in the short-distance Wilson coefficients arise. Various NP models have been proposed to explain these anomalies (leptoquarks, partial compositeness, extended electroweak gauge sector...). In order to make a selection among these models, it is clear that more data from LHCb and Belle-II are needed in the coming years and that they will remain a topic of intense discussion both experimentally and theoretically.

\section{Acknowledgments}

This project has received funding from the European Unions Horizon 2020 research and innovation programme under the Marie Sklodowska-Curie grant agreements No 690575 and 674896. 


\section{References}

[1] S. Bifani, S. Descotes-Genon, A. Romero Vidal and M. H. Schune, J. Phys. G 46 (2019) no.2, 023001

[2] Y. Amhis et al. [HFLAV Collaboration], Eur. Phys. J. C 77 (2017) no.12, 895.

[3] J. A. Bailey et al. [MILC Collaboration], Phys. Rev. D 92 (2015) no.3, 034506; H. Na et al. [HPQCD Collaboration], Phys. Rev. D 92 (2015) no.5, 054510, [Err: Phys. Rev. D 93 (2016) no.11, 119906].

[4] S. Fajfer, J. F. Kamenik and I. Nisandžić, Phys. Rev. D 85 (2012) 094025

[5] D. Bečirević et al., arXiv:1602.03030 [hep-ph] and arXiv:1907.02257 [hep-ph].

[6] P. Böer et al., JHEP 1806 (2018) 155 and arXiv:1907.12554 [hep-ph].

[7] X. Q. Li, Y. D. Yang and X. Zhang, JHEP 1608 (2016) 054; R. Alonso, B. Grinstein and J. Martin Camalich, Phys. Rev. Lett. 118 (2017) 081802; A. G. Akeroyd and C. H. Chen, Phys. Rev. D 96 (2017) 075011.

[8] M. Blanke et al., Phys. Rev. D 99 (2019) no.7, 075006 and arXiv:1905.08253 [hep-ph].

[9] S. Descotes-Genon et al., JHEP 1301 (2013) 048 and JHEP 1305 (2013) 137.

[10] A. Khodjamirian et al., JHEP 1009 (2010) 089.

[11] A. Bharucha, D. M. Straub and R. Zwicky, JHEP 1608 (2016) 098; N. Gubernari, A. Kokulu and D. van Dyk, JHEP 1901 (2019) 150

[12] S. Descotes-Genon, A. Khodjamirian and J. Virto, arXiv:1908.02267 [hep-ph].

[13] B. Capdevila et al., JHEP 1610 (2016) 075

[14] F. Kruger and J. Matias, Phys. Rev. D 71 (2005) 094009. J. Matias et al., JHEP 1204 (2012) 104. C. Bobeth, G. Hiller and D. van Dyk, JHEP 1007 (2010) 098.

[15] T. Blake et al., Eur. Phys. J. C 78 (2018) 453; C. Bobeth et al., Eur. Phys. J. C 78 (2018) 451

[16] A. Ali, G. F. Giudice and T. Mannel, Z. Phys. C 67 (1995) 417. G. Hiller and F. Kruger, Phys. Rev. D 69 (2004) 074020. S. Descotes-Genon et al., JHEP 1106 (2011) 099. W. Altmannshofer, P. Paradisi and D. M. Straub, JHEP 1204 (2012) 008. C. Bobeth, G. Hiller and D. van Dyk, Phys. Rev. D 87 (2013) 034016.

[17] S. Descotes-Genon, J. Matias and J. Virto, Phys. Rev. D 88 (2013) 074002.

[18] W. Altmannshofer and D. M. Straub, Eur. Phys. J. C 73 (2013) 2646; F. Beaujean, C. Bobeth and D. van Dyk, Eur. Phys. J. C 74 (2014) 2897 [Err: Eur. Phys. J. C 74 (2014) 3179].

[19] S. Descotes-Genon et al, JHEP 1606 (2016) 092; B. Capdevila et al., JHEP 1801 (2018) 093; M. Algueró et al., Phys. Rev. D 99 (2019) no.7, 075017

[20] M. Algueró et al., arXiv:1903.09578 [hep-ph].

[21] J. Aebischer et al., arXiv:1903.10434 [hep-ph]; M. Ciuchini et al., arXiv:1903.09632 [hep-ph]; A. Arbey et al., Phys. Rev. D 100 (2019) no.1, 015045

[22] M. Algueró et al., JHEP 1907 (2019) 096

[23] B. Capdevila et al., Phys. Rev. Lett. 120 (2018) no.18, 181802

[24] A. Crivellin et al., Phys. Rev. Lett. 122 (2019) no.1, 011805

[25] P. Böer, T. Feldmann and D. van Dyk, JHEP 1501 (2015) 155; S. Descotes-Genon and M. Novoa Brunet, JHEP 1906 (2019) 136

[26] D. Buttazzo et al., JHEP 1711 (2017) 044 